\title{
Perfil epidemiológico dos pacientes com fratura da coluna cervical tratados cirurgicamente no serviço de neurocirurgia do Hospital de Base do Distrito Federal (Brasília, Brasil)
}

\section{Epidemiological Profile of Patients with Cervical Spine Fractures Treated Surgically in the Neurosurgery Service at Hospital de Base do Distrito Federal (Brasilia, Brazil)}

\author{
Cléciton Braga Tavares ${ }^{1}$ Emerson Brandão Sousa ${ }^{1}$ Igor Brenno Campbell Borges ${ }^{1}$ \\ Amauri Araújo Godinho Júnior ${ }^{2}$ Nelson Geraldo Freire Neto ${ }^{2}$ \\ 2 Médico Neurocirurgião e Preceptor do Programa de Residência \\ Address for correspondence Cléciton Braga Tavares, Condomínio \\ Play Ilhotas, Torre Happy, Apt 14, Ilhotas, Teresina - Piaui, Rua Gov \\ Tibério Nunes CEP: 64015-040 (e-mail: bragatavares@yahoo.com.br).
}

\footnotetext{
${ }^{1}$ Médico Residente de Neurocirurgia do Hospital de Base do Distrito Federal (HBDF), Brasília, Brasil Médica em Neurocirurgia do HBDF, Brasília, Brasil
}

Arq Bras Neurocir 2016;35:1-7.

\section{Resumo \\ Palavras-Chave \\ - estudo epidemiológico \\ - traumatismo da coluna cervical, epidemiologia \\ - coluna vertebral \\ - traumatismos da coluna vertebral, epidemiologia \\ - traumatismos da coluna vertebral, cirurgia \\ - vértebras cervicais, cirurgia}

Introdução As fraturas cervicais correspondem a um grande espectro de lesões. Em alguns trabalhos a coluna cervical é o segmento mais acometido nas fraturas vertebrais, representando cerca de $45-60 \%$ de todos os casos. Apresentamos o perfil epidemiológico de 48 pacientes com fratura traumática da coluna cervical tratados cirurgicamente no serviço de neurocirurgia do Hospital de Base do Distrito Federal. Métodos Trata-se de um trabalho retrospectivo, baseado na revisão de prontuários médicos, de pacientes com fratura da coluna cervical operados no período de julho de 2007 a julho de 2012.

Resultados Predomínio do sexo masculino (89,5\%); a faixa etária mais comum é de 20-40 anos (50\%); idade média do sexo masculino é de 36,6 anos e do feminino, 19,8 anos (teste t: $p=0,04)$; vértebra mais fraturada: $C 5$ (53,3\%); $54,1 \%$ dos indivíduos apresentam algum déficit neurológico; fratura do tipo B é mais comum na coluna cervical (62,2\%); mecanismos do trauma: acidente automobilístico (41,6\%), queda de altura $(20,8 \%)$, acidente motociclístico $(12,5 \%)$, outros $(25,1 \%)$; déficit neurológico da cervical alta (zero) e subaxial de $57,7 \%(p=0,052)$; déficit neurológico masculino de $53,4 \%$ e feminino de $60 \%$ ( $p>0,05$ ); déficit neurológico do tipo $A$ de $71,4 \%$, do tipo $B$ de $55,5 \%$, e do tipo $C$ de $54,5 \%$ ( $p>0,05)$.

Conclusão A maioria dos pacientes era do sexo masculino e da faixa etária entre 20 e 40 anos de idade, sendo as mulheres mais jovens que os homens. Cerca de $54,1 \%$ dos pacientes apresentavam déficit neurológico à admissão hospitalar e tinham C5 como received

September 9, 2012

accepted

August 7, 2015

published online

October 28, 2015
DOI http://dx.doi.org/

10.1055/s-0035-1564826. ISSN 0103-5355.
Copyright $(2016$ by Thieme Publicações License terms Ltda, Rio de Janeiro, Brazil
(요 (1) $\Theta \circledast$ 


\section{Abstract}

\author{
Keywords \\ - epidemiological \\ study \\ - cervical spine \\ trauma, \\ epidemiology \\ - spinal cord \\ - spinal injuries, \\ epidemiology \\ - spinal injuries, \\ surgery \\ - cervical spine, \\ surgery
}

principal vértebra fraturada. A fratura tipo distração (tipo B da AO) foi a mais encontrada. O principal mecanismo do trauma foram os acidentes de trânsito seguido pelas quedas de altura.

Introduction The cervical fractures represent a wide spectrum of injuries. In some works, the cervical spine is the segment most affected vertebral fractures, representing $\sim 45-60 \%$ of all cases. We present the epidemiological profile of forty-eight patients with traumatic cervical spine fracture surgically treated in the neurosurgery service at the Hospital of the Federal District.

Methods This was a retrospective study, based on a review of medical records of patients with cervical spine fracture surgery from July 2007 to July 2012.

Results Predominance of males (89.5\%), the most common age group is $20-40$ years (50\%), mean age: males (36.6 years) and females (19.8 years) (t-test $p=0.04$ ); fractured vertebra: C5 (53.3\%), $54.1 \%$ of subjects have a neurological deficit, fracture type $B$ is more common in the cervical spine (62.2\%), mechanism of injury: motor vehicle accidents (41.6\%), fall (20.8\%), motorcycle (12.5\%), other (25.1\%), neurological deficit: high cervical 0 cases, subaxial 57.7\% ( $p=0.052)$; neurological deficit: male $53.4 \%$, female $60 \%$ ( $p>0.05$ ); neurological deficit: $71.4 \%$ Type A, Type B 55.5\% 54.5\% Type C ( $\mathrm{p}>0.05)$.

Conclusion Most patients were male and the age group between 20-40 years of age, with younger women than men. Approximately $54.1 \%$ of the patients had neurologic deficit on admission and had as main fractured vertebra $C 5$. The distraction fractures (AO Type B) was found most frequently. The main mechanism of injury were traffic accidents followed by falls from height.

\section{Introdução}

A coluna cervical pode ser dividida em alta (côndilos C1 e C2) e subaxial ou baixa (vértebras C3 a C7). ${ }^{1}$

A coluna cervical alta apresenta características anatômicas diferentes das demais vértebras da coluna cervical: não existe disco intervertebral, e a estabilidade é fornecida por um complexo sistema ligamentar que conecta o atlas e o áxis à base do occipital. As lesões cervicais altas são infrequentes, e a sua exata incidência não é bem conhecida devido à sua ocorrência em vítimas fatais dos acidentes de trânsito. As lesões neurológicas são raras devido ao maior diâmetro do canal raquidiano em relação à medula espinhal. No entanto, as lesões neurológicas neste nível podem ser fatais. ${ }^{1}$

As fraturas da coluna cervical subaxial correspondem a um grande espectro de lesões. Grande porcentagem destas não tem sido diagnosticada no atendimento inicial dos pacientes, enfatizando a necessidade de um exame clínico detalhado e da visualização de toda a extensão da coluna cervical nos exames de imagem dos pacientes com potencial lesão deste segmento. ${ }^{1}$

Em alguns estudos a coluna cervical é o segmento mais acometido nas fraturas da coluna vertebral, representando cerca de $45-60 \%$ de todos os casos, com elevada taxa de morbidade e mortalidade. ${ }^{2}$

Apresentamos o perfil epidemiológico e os fatores de risco para déficit neurológico de 48 pacientes com fratura traumática da coluna cervical tratados cirurgicamente no serviço de neurocirurgia do Hospital de Base do Distrito Federal. É um serviço de referência, da capital federal e do centro-oeste brasileiro, no tratamento do traumatismo raquimedular, com realização média de 100 cirurgias por ano para correção de fraturas vertebrais. Os dados levantados são importantes do ponto de vista de saúde pública e gestão de recursos.

\section{Materiais e Métodos}

Trata-se de um trabalho retrospectivo, baseado na revisão de prontuários médicos, de pacientes com fratura da coluna cervical tratados cirurgicamente no serviço de neurocirurgia do Hospital de Base do Distrito Federal em Brasília, no período de julho de 2007 a julho de 2012.

Neste intervalo de tempo, foram operados no hospital 515 pacientes com fratura vertebral, sendo 200 cervicais (31\%) e 315 torácicas e lombares (69\%).

Os dados levantados foram: faixa etária, sexo, mecanismo do trauma, vértebras fraturadas, tipo de fratura (baseado na classificação da Magerl/AO) e déficit neurológico (baseado na classificação da ASIA - American Spinal Injury Association/ Frankel). Os prontuários que não apresentavam todos estes dados, no relatório de alta hospitalar, foram excluídos do trabalho - ao todo, os de 152 indivíduos.

Os pacientes foram agrupados em cinco faixas etárias: $0-10, \quad 10-20,20-40,40-60$ e acima de 60 anos. Os mecanismos do trauma foram divididos em dez grupos: acidentes automobilísticos, acidentes motociclísticos, queda 
de altura, queda da própria altura, lesão por arma de fogo, mergulho em águas rasas, acidentes ciclísticos, acidentes com máquina agrícola, agressão física e queda de material pesado sobre o corpo.

A classificação das fraturas utilizada foi a de Margerl et al, ${ }^{3}$ adotada pelo Grupo AO, que agrupa as fraturas em três grandes tipos: (A) lesões por compressão do corpo vertebral, (B) lesões por distração dos elementos anterior e/ou posterior e (C) lesões tipo A ou B com rotação e luxações complexas. Cada tipo, por sua vez, é subdividido em três subtipos: 1,2 e 3. E cada subtipo é dividido em três subgrupos: 1,2 e 3. No presente estudo, optou-se por restringir a classificação aos tipos.

A avaliação da deficiência neurológica foi baseada na escala de Frankel et al, ${ }^{4}$ que foi modificada pela ASIA e consiste em cinco graus de incapacidade: (A) lesão completa, não existe função motora ou sensitiva nos segmentos sacrais S4-S5; (B) lesão incompleta, preservação da sensibilidade e perda total da força motora abaixo do nível neurológico, estendendo-se até os segmentos sacrais S4-S5; (C) lesão incompleta, preservação da função motora abaixo do nível neurológico, a maioria dos músculos-chave possuem força de grau 1 ou 2; (D) lesão incompleta, preservação da função motora abaixo do nível neurológico, a maioria dos músculoschave possuem força de grau 3 ou 4; e (E) sensibilidade e motricidade normais, força de grau 5 .

A gradação da força muscular foi realizada de acordo com o método do Medical Research Council, desta forma: grau 0, paralisia total; grau 1, contração fracamente detectável; grau 2 , força insuficiente para atuar contra gravidade; grau 3 , força suficiente para atuar contra a gravidade; Grau 4: Força presente, porém ainda não é normal; Grau 5: Força normal. ${ }^{5}$

As informações foram compiladas em uma planilha do programa Excel, perfazendo um total de 48 indivíduos $(n=48)$. Todos os dados foram analisados pelo software Statistical Package for the Social Sciences (SPSS 18.0). As associações foram avaliadas com teste do qui-quadrado e

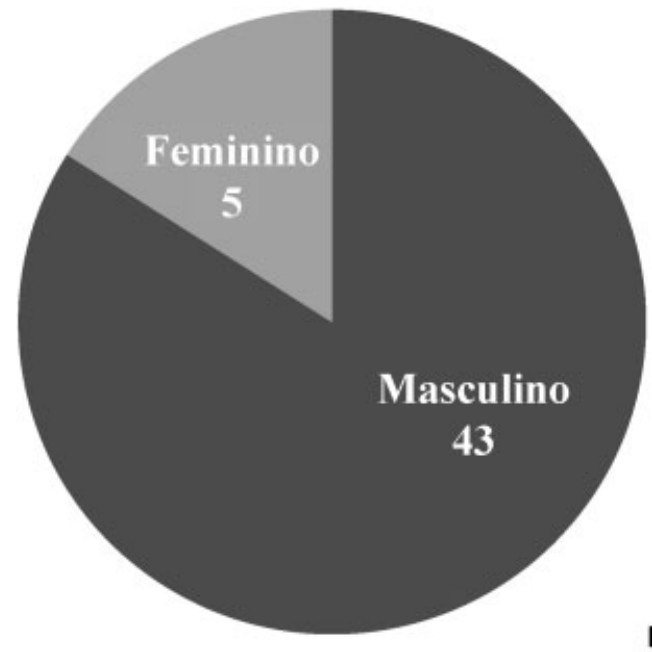

n 48

Fig. 1 Distribuição de acordo com o sexo dos pacientes com fraturas cervicais tratadas cirurgicamente no serviço de neurocirurgia do HBDF entre julho de 2007 e julho de 2012. Fonte: Serviço de Arquivo Médico do Hospital de Base do Distrito Federal, Brasília, Brasil.

com o teste $\mathrm{t}$ para comparação de médias aritméticas. Um valor de $p<0,05$ foi considerado estatisticamente significativo.

\section{Discussão}

A maioria dos pacientes deste estudo foi do sexo masculino (8,6:1), com predomínio na faixa etária de 20-40 anos de idade. A média de idade entre as mulheres com fratura vertebral (19,8 anos) foi menor que a dos homens (36,6 anos), diferença estatisticamente significativa, de acordo com o teste $\mathrm{t}$ para comparação entre médias aritméticas (-Figs. 1, 2 e 3). Os dados são condizentes com a literatura e explicados pelo fato de os homens jovens se exporem mais às atividades laborativas e recreativas de risco e

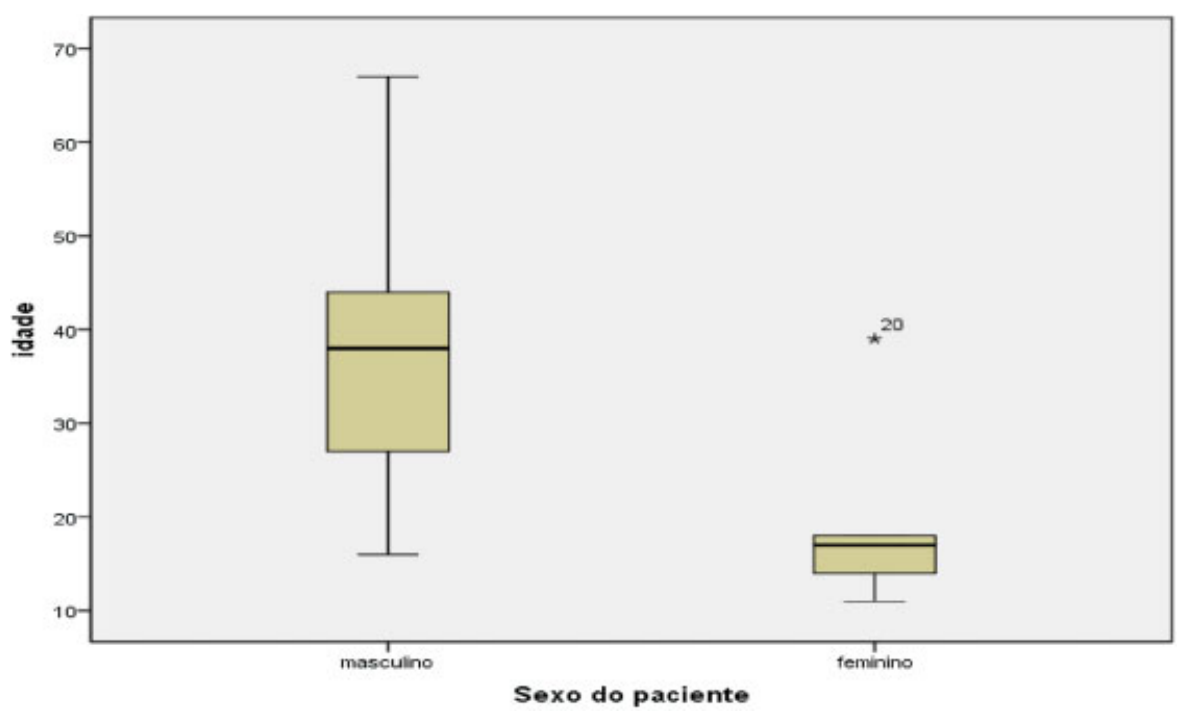

Fig. 2 Distribuição de acordo com o sexo e a idade dos pacientes com fraturas cervicais tratadas cirurgicamente no serviço de neurocirurgia do HBDF entre julho de 2007 e julho de 2012. Idade média geral: 34,8 anos (11-67 anos); sexo masculino: 36,6 anos; sexo feminino: 19,8 anos; (teste t: $p=0,04)$. Fonte: Serviço de Arquivo Médico do Hospital de Base do Distrito Federal, Brasília, Brasil. 
4 Perfil epidemiológico de fraturas cervicais tratadas no HBDF de 2007 a 2012 Tavares et al.

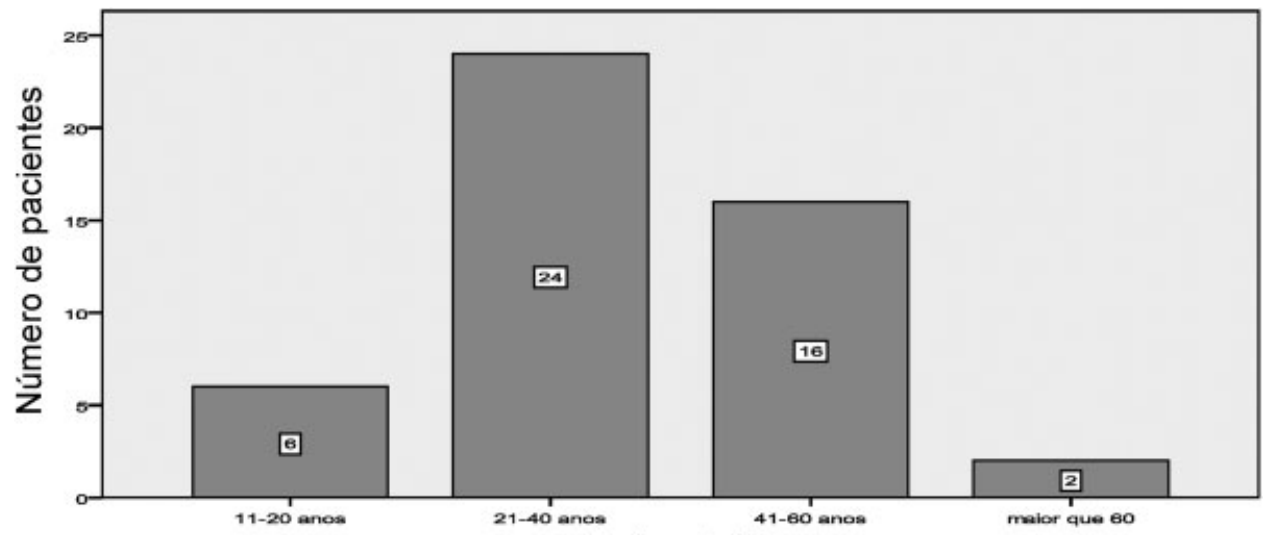

Faixa et ária

Fig. 3 Distribuição de acordo com a faixa etária dos pacientes com fraturas cervicais tratadas cirurgicamente no serviço de neurocirurgia do HBDF entre julho de 2007 e julho de $2012(n=48)$. Fonte: Serviço de Arquivo Médico do Hospital de Base do Distrito Federal, Brasília, Brasil.

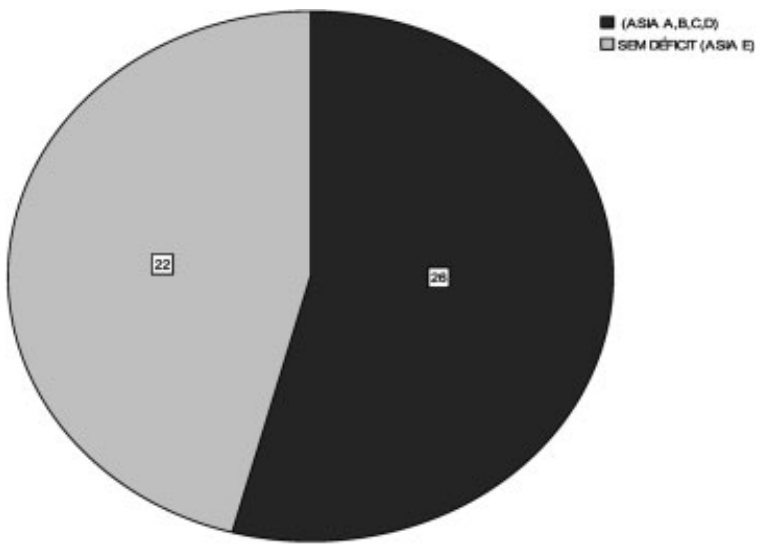

Fig. 4 Distribuição de acordo com o déficit neurológico dos pacientes com fraturas cervicais tratadas cirurgicamente no serviço de neurocirurgia do HBDF entre julho de 2007 e julho de $2012(n=48)$. Abreviações: ASIA, American Spinal Injury Association. Fonte: Serviço de Arquivo Médico do Hospital de Base do Distrito Federal, Brasília, Brasil. serem os principais envolvidos em comportamentos violentos. $^{1,6-8}$

Cerca de 54,1\% dos pacientes apresentaram algum déficit neurológico à admissão hospitalar, de acordo com a classificação da ASIA/Frankel, (-Fig. 4). Santos et al ${ }^{2}$ encontraram $67 \%$ de indivíduos com fratura cervical e déficit neurológico, sendo a maioria $(34,6 \%)$ com lesão completa. Vaccaro et $\mathrm{al}^{9}$ encontraram $40 \%$ de déficit completo, $40 \%$ de incompleto e $20 \%$ de pacientes sem lesão neurológica entre indivíduos com fratura cervical.

A vértebra cervical mais fraturada foi C5 (-Fig. 5). Os dados são semelhantes aos encontrados por Feng et al, ${ }^{7}$ nos quais a maioria das fraturas vertebrais cervicais se encontrava em C4-C6, provavelmente devido à maior amplitude de movimentos durante a flexão-extensão que este segmento apresenta. 1

A maior parte das fraturas tratadas cirurgicamente foi do tipo distração (tipo B da AO) ( - Fig. 6). Segundo Holtz e Levi, a hiperflexão-translação e a hiperflexão-compressão são responsáveis por cerca de $60 \%$ das fraturas cervicais. ${ }^{10}$ Isto, associado ao fato de que as facetas articulares têm uma angulação aproximada de $45^{\circ}$, favorece a presença da

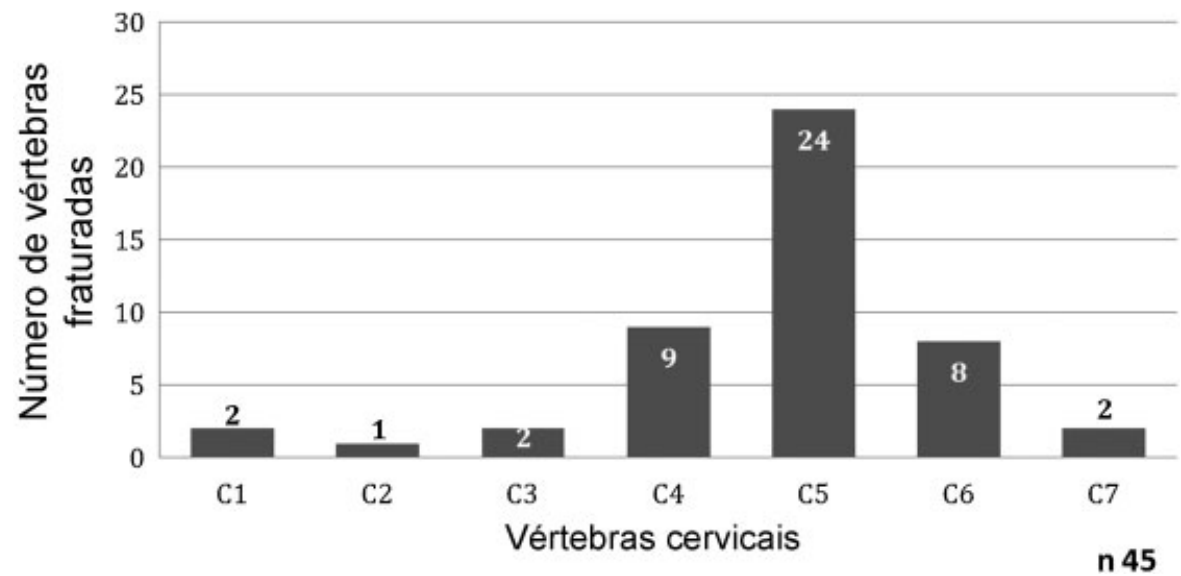

Fig. 5 Número de vértebras cervicais fraturadas e tratadas cirurgicamente no serviço de neurocirurgia do HBDF entre julho de 2007 e julho de 2012. Fonte: Serviço de Arquivo Médico do Hospital de Base do Distrito Federal, Brasília, Brasil. 


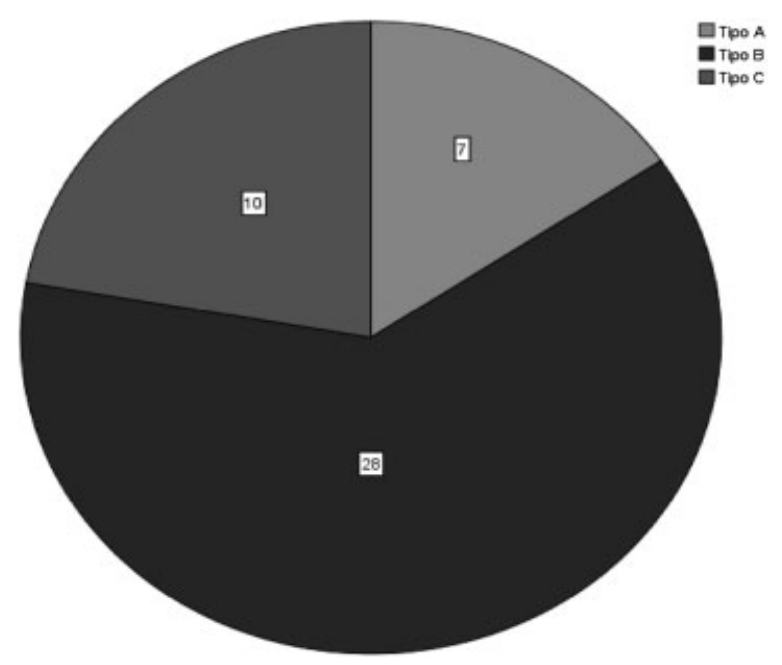

Fig. 6 Distribuição de acordo com a classificação das fraturas cervicais tratadas cirurgicamente no serviço de neurocirurgia do HBDF entre julho de 2007 e julho de 2012 ( $n=45)$. Fonte: Serviço de Arquivo Médico do Hospital de Base do Distrito Federal, Brasília, Brasil.

distração entre as lesões cervicais. ${ }^{1}$ Talvez esta seja a explicação das fraturas do tipo B serem mais frequentes neste nível.

Os acidentes automobilísticos foram a principal causa de fraturas cervicais, seguidos por quedas de altura e acidentes motociclísticos (-Fig. 7). Achado semelhante ao do trabalho de Frielingsdorf et al. ${ }^{11}$ Já o trabalho de Santos et $\mathrm{al}^{2}$ evidenciou um predomínio da queda de altura sobre os outros mecanismos do trauma. Estes achados dependem das características demográficas, habitacionais e principalmente socioeconômicas de cada região. ${ }^{12-14}$

As fraturas cervicais altas apresentaram menor número de pacientes com lesão neurológica do que as fraturas subaxiais, e a diferença entre elas foi muito próxima de ser estatisticamente significativa (-Tabela 1 ). No entanto, o trabalho de Santos et al, ${ }^{2}$ que utilizou uma amostra maior, demonstrou matematicamente esta diferença. Entre as fraturas cervicais, o sexo, a faixa etária, o mecanismo do trauma, o tipo de fratura segundo a classificação da $\mathrm{AO}$ e a vértebra cervical acometida não foram fatores de risco para lesão neurológica, uma vez que as diferenças observadas nas - Tabelas 1 a 6 não foram estatisticamente significativas.

\section{Conclusão}

A maioria dos pacientes com fraturas cervicais tratadas cirurgicamente, no serviço de Neurocirurgia do Hospital de Base do Distrito Federal no período de julho de 2007 a julho de 2012, era do sexo masculino e da faixa etária compreendida entre 20 e 40 anos de idade, sendo as mulheres mais jovens que os homens.

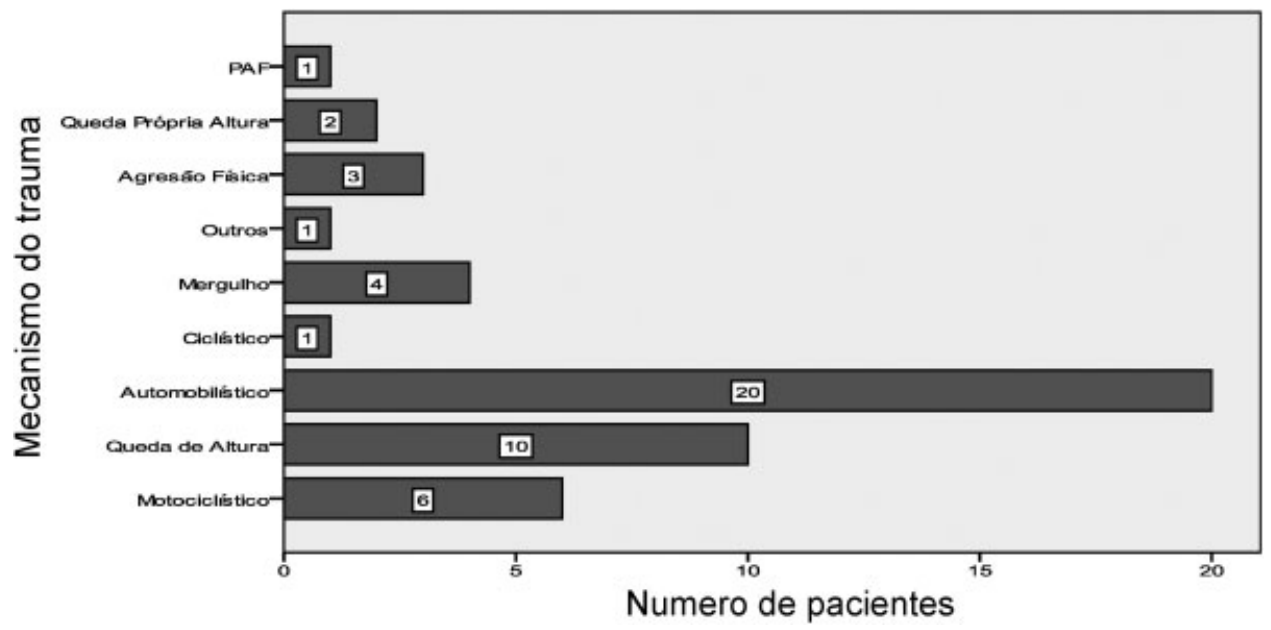

Fig. 7 Distribuição de acordo com o mecanismo do trauma das fraturas cervicais tratadas cirurgicamente no serviço de neurocirurgia do HBDF entre julho de 2007 e julho de 2012. Fonte: Serviço de Arquivo Médico do Hospital de Base do Distrito Federal, Brasília, Brasil.

Tabela 1 Número de pacientes com déficit neurológico de acordo com o segmento vertebral da coluna cervical acometido

\begin{tabular}{|l|l|l|l|}
\hline \multirow{2}{*}{ Coluna cervical } & Déficit neurológico & \multirow{2}{*}{ Total } \\
\cline { 2 - 4 } & $\begin{array}{l}\text { com déficit } \\
\text { (ASIA A, B, C, D) }\end{array}$ & $\begin{array}{l}\text { sem déficit } \\
\text { (ASIA E) }\end{array}$ & 3 \\
\hline Alta & 0 & 3 & 45 \\
\hline Subaxial & 26 & 19 & 48 \\
\hline Total & 26 & 22 & \\
\hline
\end{tabular}

$\mathrm{n}=48 ; p=0,052$.

Abreviações: ASIA, American Spinal Injury Association.

Fonte: Serviço de Arquivo Médico do Hospital de Base do Distrito Federal, Brasília, Brasil. 
6 Perfil epidemiológico de fraturas cervicais tratadas no HBDF de 2007 a 2012 Tavares et al.

Tabela 2 Número de pacientes com déficit neurológico de acordo com a vértebra cervical fraturada

\begin{tabular}{|l|l|l|l|}
\hline \multirow{2}{*}{ Vértebra fraturada } & Déficit neurológico & \multirow{2}{*}{ Total } \\
\cline { 2 - 4 } & $\begin{array}{l}\text { com déficit } \\
\text { (ASIA A, B, C, D) }\end{array}$ & $\begin{array}{l}\text { sem déficit } \\
\text { (ASIA E) }\end{array}$ & 2 \\
\hline C1 & 0 & 2 & 1 \\
\hline C2 & 0 & 1 & 2 \\
\hline C3 & 1 & 1 & 9 \\
\hline C4 & 4 & 5 & 24 \\
\hline C5 & 16 & 8 & 8 \\
\hline C6 & 4 & 4 & 1 \\
\hline C7 & 1 & 1 & 2 \\
\hline Total & 26 & 22 & 48 \\
\hline
\end{tabular}

$\mathrm{N}=48 ; p=0,484$

Abreviações: AO, Arbeitsgemeinschaft für Osteosynthesefragen (Associação para o Estudo de Fixação Interna); ASIA, American Spinal Injury Association.

Tabela 3 Número de pacientes com déficit neurológico de acordo com o tipo de fratura cervical subaxial (classificação AO)

\begin{tabular}{|l|l|l|l|}
\hline \multirow{2}{*}{ Classificação da AO } & Déficit neurológico & \multirow{2}{*}{ Total } \\
\cline { 2 - 4 } & $\begin{array}{l}\text { com déficit } \\
\text { (ASIA A, B, C, D) }\end{array}$ & $\begin{array}{l}\text { sem déficit } \\
\text { (ASIA E) }\end{array}$ & 2 \\
\hline A & 5 & 2 & 7 \\
\hline B & 15 & 13 & 28 \\
\hline C & 6 & 4 & 10 \\
\hline Total & 26 & 19 & 45 \\
\hline
\end{tabular}

$\mathrm{n}=45 ; p=0,685$.

Abreviações: AO, Arbeitsgemeinschaft für Osteosynthesefragen (Associação para o Estudo de Fixação Interna); ASIA, American Spinal Injury Association.

Fonte: Serviço de Arquivo Médico do Hospital de Base do Distrito Federal, Brasília, Brasil.

Tabela 4 Número de pacientes com fratura cervical e déficit neurológico de acordo com a faixa etária

\begin{tabular}{|l|l|l|l|}
\hline \multirow{2}{*}{ Faixa etária } & Déficit neurológico & \multirow{2}{*}{ Total } \\
\cline { 2 - 4 } & $\begin{array}{l}\text { com déficit } \\
\text { (ASIA A, B, C, D) }\end{array}$ & $\begin{array}{l}\text { sem déficit } \\
\text { (ASIA E) }\end{array}$ & 6 \\
\hline $11-20$ & 5 & 1 & 14 \\
\hline $21-40$ & 10 & 7 & 24 \\
\hline $41-60$ & 9 & 0 & 16 \\
\hline$>60$ & 2 & 22 & 2 \\
\hline Total & 26 & & 48 \\
\hline
\end{tabular}

$\mathrm{N}=48 ; p=0,152$.

Abreviações: ASIA, American Spinal Injury Association.

Fonte: Serviço de Arquivo Médico do Hospital de Base do Distrito Federal, Brasília, Brasil.

Tabela 5 Número de pacientes com fratura cervical e déficit neurológico de acordo com o sexo

\begin{tabular}{|l|l|l|l|}
\hline \multirow{2}{*}{ Sexo do paciente } & \multicolumn{2}{|l|}{ Déficit neurológico } & \multirow{2}{*}{ Total } \\
\cline { 2 - 4 } & $\begin{array}{l}\text { com déficit } \\
\text { (ASIA A, B, C, D) }\end{array}$ & $\begin{array}{l}\text { sem déficit } \\
\text { (ASIA E) }\end{array}$ & 43 \\
\hline Masculino & 23 & 20 & 5 \\
\hline Feminino & 3 & 2 & 22 \\
\hline Total & 26 & 2 & 48 \\
\hline
\end{tabular}

$\mathrm{N}=48 ; p=0,782$.

Abreviações: ASIA, American Spinal Injury Association.

Fonte: Serviço de Arquivo Médico do Hospital de Base do Distrito Federal, Brasília, Brasil. 
Tabela 6 Número de pacientes com fratura cervical e déficit neurológico de acordo com o mecanismo do trauma

\begin{tabular}{|l|l|l|l|}
\hline \multirow{2}{*}{ Mecanismo do trauma } & \multicolumn{2}{|l|}{ Déficit neurológico } & \multirow{2}{*}{ Total } \\
\cline { 2 - 4 } & $\begin{array}{l}\text { com déficit } \\
\text { (ASIA A, B, C, D) }\end{array}$ & $\begin{array}{l}\text { sem déficit } \\
\text { (ASIA E) }\end{array}$ & 6 \\
\hline Acidente motociclístico & 2 & 4 & 10 \\
\hline Queda de altura & 7 & 3 & 20 \\
\hline Acidente automobilístico & 12 & 8 & 1 \\
\hline Acidente ciclístico & 0 & 1 & 4 \\
\hline Mergulho & 2 & 2 & 3 \\
\hline Agressão física & 0 & 3 & 2 \\
\hline Queda da própria altura & 1 & 1 & 1 \\
\hline PAF & 1 & 0 & 1 \\
\hline Outros & 1 & 0 & 48 \\
\hline Total & 26 & 22 & \\
\hline
\end{tabular}

$\mathrm{n}=48 ; p=0,360$

Abreviações: ASIA, American Spinal Injury Association; PAF, perfuração por arma de fogo.

Fonte: Serviço de Arquivo Médico do Hospital de Base do Distrito Federal, Brasília, Brasil.

Dos pacientes, 54,1\% apresentavam déficit neurológico à admissão hospitalar e 53,3\% tinham C5 como principal vértebra fraturada.

A fratura do tipo distração (tipo $\mathrm{B}$ da $\mathrm{AO}$ ) foi a mais encontrada. $O$ principal mecanismo do trauma foram os acidentes de trânsito seguidos pelas quedas de altura.

A importância deste estudo está principalmente na demonstração da distribuição de acordo com o mecanismo do trauma das fraturas cervicais tratadas no HBDF. Os resultados encontrados devem ser levados ao conhecimento dos órgãos responsáveis pelo controle do trânsito no país, para que campanhas educativas sejam elaboradas com auxílio dos gestores da área da saúde.

\section{Agradecimentos}

Quero aproveitar e agradecer a minha mãe Neusa por todo o esforço, carinho e dedicação na criação e estímulo educacional deste jovem piauiense, ao meu pai Diaslano, que me protege dos céus, e também a minha futura esposa Francisca pelo amor dispensado e pela paciência em me aturar e apoiar ao longo destes 5 anos de residência.

\section{Referências}

1 Defino HLA. Lesões Traumáticas da Coluna Vertebral. São Paulo: Bevilacqua Editora; 2005

2 Santos EAS, Filho WJ, Possatti LL, Bittencourt LR, Fontoura EA, Botelho RV. Epidemiology of severe cervical spinal trauma in the north area of São Paulo City: a 10-year prospective study. Clinical article. J Neurosurg Spine 2009;11(1):34-41

3 Magerl F, Aebi M, Gertzbein SD, Harms J, Nazarian S. A comprehensive classification of thoracic and lumbar injuries. Eur Spine J 1994;3(4):184-201

4 Frankel HL, Hancock DO, Hyslop G, et al. The value of postural reduction in the initial management of closed injuries of the spine with paraplegia and tetraplegia. I. Paraplegia 1969;7(3):179-192

5 Campbell WW. Dejong O Exame Neurológico. Rio de Janeiro: Guanabara Koogan; 2007

6 Campos MF, Ribeiro AT, Listik S, Pereira CA, Sobrinho JA, Rapoport A. Epidemiologia do Traumatismo da Coluna Vertebral no Serviço de Neurocirurgia do Hospital Heliópolis, São Paulo, Brasil. Rev Col Bras Cir 2008;35(2):88-93

7 Ning GZ, Yu TQ, Feng SQ, et al. Epidemiology of traumatic spinal cord injury in Tianjin, China. Spinal Cord 2011;49(3):386-390

8 Vasconcelos ECLM, Riberto M. Caracterização clínica e das situações de fratura da coluna vertebral no município de ribeirão preto, propostas para um programa de prevenção do trauma raquimedular. Coluna 2011;10(1):40-43

9 Vaccaro AR, Daugherty RJ, Sheehan TP, et al. Neurologic outcome of early versus late surgery for cervical spinal cord injury. Spine 1997;22(22):2609-2613

10 Holtz A, Levi R. Spinal Cor Injury. Oxford University Press; 2010

11 Fielingsdorf K, Dunn RN. Cervical spine injury outcome-a review of 101 cases treated in a tertiary referral unit. S Afr Med J 2007; 97(3):203-207

12 Sekhon LH, Fehlings MG. Epidemiology, demographics, and pathophysiology of acute spinal cord injury. Spine 2001;26(24, Suppl):S2-S12

13 Stover SL, Fine PR. The epidemiology and economics of spinal cord injury. Paraplegia 1987;25(3):225-228

14 Rhee P, Kuncir EJ, Johnson L, et al. Cervical spine injury is highly dependent on the mechanism of injury following blunt and penetrating assault. J Trauma 2006;61(5):1166-1170 